\title{
Recent star formation at low metallicities. The star-forming region NGC 346/N66 in the Small Magellanic Cloud from near-infrared VLT/ISAAC observations ${ }^{\star}, \star \star$
}

\author{
D. A. Gouliermis ${ }^{1}$, J. M. Bestenlehner ${ }^{1,2}$, W. Brandner ${ }^{1}$, and T. Henning ${ }^{1}$ \\ 1 Max-Planck-Institut für Astronomie, Königstuhl 17, 69117 Heidelberg, Germany \\ e-mail: dgoulier@mpia-hd.mpg.de \\ 2 Armagh Observatory, College Hill, Armagh BT61 9DG, UK
}

Received 29 March 2009 / Accepted 27 February 2010

\begin{abstract}
Context. The emission nebula N66 is the brightest H II region in the Small Magellanic Cloud (SMC), the stellar association NGC 346 being located at its center. The youthfulness of the region NGC 346/N66 is well documented by studies of the gas and dust emission, and the detection in the optical of a rich sample of pre-main sequence (PMS) stars, and in the mid- and far-IR of young stellar objects (YSOs). However, a comprehensive study of this region has not been performed in the near-IR that would bridge the gap between previous surveys.

Aims. We perform a photometric analysis on deep, seeing-limited near-IR VLT images of the region NGC 346/N66 and a nearby control field of the SMC to locate the centers of active high- and intermediate-mass star formation by identifying near-IR bright objects as candidate stellar sources under formation.

Methods. We use archival imaging data obtained with the high-resolution camera ISAAC at VLT of NGC 346/N66 to construct the near-IR color-magnitude (CMD) and color-color diagrams (C-CD) of all detected sources. We investigate the nature of all stellar populations in the observed CMDs, and we identify all stellar sources that show significant near-IR excess emission in the observed C-CD. We, thus, select the most likely young stellar sources.

Results. Based on their near-IR colors, we select 263 candidate young stellar sources. This sample comprises a variety of objects, such as intermediate-mass PMS and Herbig Ae/Be stars and possible massive YSOs, providing original near-IR colors for each of them. The spatial distribution of the selected candidate sources indicates that they are located along the dusty filamentary structures of N66 seen in mid- and far-IR dust emission and agrees very well with that of previously detected candidate YSOs and PMS stars. Conclusions. Our study provides an original accurate set of near-IR colors for candidate young stellar sources. This provides significant information about the star formation process in NGC 346/N66, but does not establish the types of these objects, which requires the construction of complete spectral energy distributions for individual sources from multiwavelength data. This would be an important follow-up study to that presented here.
\end{abstract}

Key words. stars: pre-main-sequence - Magellanic Clouds - open clusters and associations: individual: NGC 346 - HII regions ISM: individual objects: LHA 115-N66 - catalogs

\section{Introduction}

Located in the stellar constellation Tucana, the Small Magellanic Cloud (SMC), is an excellent laboratory for investigating the star formation processes and the associated chemical evolution of dwarf galaxies. Its present subsolar chemical abundance ( $Z=0.004 ; \sim 20 \%$ of solar) implies that this galaxy may have characteristics similar to those in earlier times of the evolution of the universe. Since SMC is so close to our Galaxy, it is therefore an excellent laboratory among the large collection of dwarf irregulars and blue compact galaxies for the study of resolved extragalactic stellar populations and star-forming regions. The young stellar association NGC $346\left(\right.$ RA $(J 2000)=00^{\mathrm{h}} 59^{\mathrm{m}} 18^{\mathrm{s}}$, $\left.\operatorname{DEC}(\mathrm{J} 2000)=-72^{\circ} 10^{\prime} 48^{\prime \prime}\right)$ is a large star-forming cluster

* Based on observations made with ESO Telescopes at the La Silla Paranal Observatory under program ID 063.I-0329.

$\star \star$ Table 1 is available in its entirety only in electronic form at the CDS via anonymous ftp to cdsarc.u-strasbg.fr (130.79.128.5) or via

http://cdsweb.u-strasbg.fr/cgi-bin/qcat?J/A+A/515/A56 in the SMC, located at a distance of about $60.6 \mathrm{kpc}$ from us (Hilditch et al. 2005). It is embedded in the brightest H II region of the SMC, which is referred to as LHA 115-N66 or N66 (Henize 1956). With an $\mathrm{H} \alpha$ luminosity almost 60 times higher than the star-forming region of Orion (Kennicutt 1984), N66 has a diameter of about $7^{\prime}$ corresponding to approximately $123 \mathrm{pc}$.

The star-forming region NGC 346/N66 comprises a variety of young stellar populations (Gouliermis et al. 2006). The stellar association NGC 346, located at the center of the so-called nebular "bar" of N66, hosts the largest sample of O-type stars in the entire SMC (Massey et al. 1989; Walborn et al. 2000; Evans et al. 2006). Studies based on deep imaging with the Hubble Space Telescope show that the vicinity of the whole region of NGC 346/N66 is also very rich in low- and intermediatemass PMS stars, some of which exhibit recent star formation with an age $\lesssim 5 \mathrm{Myr}$, while others belong to an older underlying population of age $\lesssim 10 \mathrm{Myr}$ (see e.g., Hennekemper et al. 2008). Additional evidence of the youthfulness of this region comes from observations with the Spitzer Space Telescope and the discovery of 111 candidate massive young stellar objects 
(YSOs) with $2 \lesssim M / M_{\odot} \lesssim 17$ (Simon et al. 2007). Nevertheless, although these objects should emit in near-IR bands, no detailed study in such wavelengths exists in the literature.

Pioneering work on the dust and gas content of NGC 346/N66 was performed by Rubio et al. (2000) and Contursi et al. (2000), who found a correlation between $\mathrm{H}_{2}$ infrared emission and $\mathrm{CO}$ lines, characteristic of a photodissociated region (PDR). A PDR develops when the far-UV radiation of the bright $\mathrm{OB}$ stars reaches the surface of the parental molecular cloud. The degree of ionization decreases outwards, and a thin barrier develops that segregates the ionized from the atomic gas. While $\mathrm{H}_{2}$ is not fully ionized behind this front but partly dissociated, $\mathrm{CO}$ molecules located little deeper in the cloud are more easily dissociated by absorbing UV photons. Rubio et al. (2000) and Contursi et al. (2000) inferred that star formation in NGC 346/N66 has taken place as a sequential process in the "bar" of $\mathrm{N} 66$, which these authors define as the oblique bright emission region extending from southeast to northwest centered on NGC 346. This process results in several embedded sources, seen as IR-emission peaks in the $2.14 \mu \mathrm{m} \mathrm{H}_{2}$ line and the ISOCAM LW2 band (5-8 $\left.\mu \mathrm{m}\right)$. These peaks are alphabetically numbered from "A" to "I" (Rubio et al. 2000; Contursi et al. 2000), the association NGC 346 itself coinciding with peak " $\mathrm{C}$ ".

Studies with the Advanced Camera for Surveys (ACS) onboard the Hubble Space Telescope (HST) have shown that the PMS stellar content of NGC 346/N66 covers a mass range in the subsolar regime. These studies suggest that recent star formation occurred around 3-5 Myr ago (Nota et al. 2006; Sabbi et al. 2007) there being an underlying older PMS population, which indicates that there were earlier star formation events that occurred about $10 \mathrm{Myr}$ ago (Hennekemper et al. 2008). The PMS population is mainly centrally concentrated apart from the association NGC 346 in a number of subclusters with clustering properties quite similar to those of Milky Way star-forming regions (Schmeja et al. 2009). Furthermore, the intermediate-age star cluster BS 90 (Bica \& Schmitt 1995) with an age of about 4.5 Gyr (Rochau et al. 2007) is also located in this part of the SMC, projected in front of N66.

This photometric study of the star-forming region NGC 346/N66 focuses on identifying young stellar sources, mainly intermediate- and high-mass PMS stars of the region, stars that have not yet started their lives on the main sequence. The PMS phase in the evolution of stars with masses up to $\sim 6 M_{\odot}$ corresponds to the time between the gravitational core collapse, which forms the protostar (on the birthline), and the ignition of hydrogen in the formed star, placing it on the ZeroAge Main Sequence (ZAMS). During this evolutionary phase, the observed radiation from the star is affected significantly by circumstellar disks of dust and gas, formed by matter infalling during the collapse of the rotating core, and surface activity. Examples of these typical PMS stars, the T Tauri stars, thus, exhibit prominent optical emission lines, which are understood to stem from chromospheric heating, periodic fluctuations in light that indicate rotating star-spots, variability, and excess broadband flux in UV and IR, and are sometimes associated with molecular outflows, winds, or accretion (e.g., Lada \& Kylafis 1991). Intermediate-mass $\left(2 \lesssim M / M_{\odot} \lesssim 10\right)$ PMS stars are called Herbig AeBe (HAeBe) stars (e.g., Perez \& Grady 1997). Being more massive analogues of T Tauri stars, they are PMS A- and B-type stars that exhibit emission lines produced by both strong stellar winds and the cocoons of remnant gas from which they collapsed. They typically contain circumstellar disks and therefore have spectral energy distributions (SEDs) of young stellar objects (YSOs) of class II. They range in age between 0.5 and $5 \mathrm{Myr}$, similar to T Tauri stars. Because of the aforementioned characteristics, PMS stars are visible in near-IR wavebands.

While extensive observations have been performed of young stellar sources in the region NGC 346/N66 at optical (Nota et al. 2006; Gouliermis et al. 2006) and mid- and far-IR (Bolatto et al. 2007; Simon et al. 2007) wavelengths, there has been no comparative investigation in the near-IR. The present study aims to close this gap in the available spectral coverage for this region by acquiring near-IR data to characterize with greater accuracy its young stellar population. This will also aid our understanding of star formation in the low-metallicity environment of the SMC. In this paper, we present our ground-based near-IR photometry derived from observations with VLT/ISAAC of the region NGC 346/N66. We present the observational material used and its reduction in Sect. 2, and we discuss the photometric process in Sect. 3. The various observed stellar populations and the corresponding stellar systems comprised in the observed field are discussed in terms of variations in the constructed colormagnitude diagrams in Sect. 4. We present the constructed nearIR color-color diagram of all detected sources and apply a selection criterion by identifying stars currently forming by means of their near-IR excess emission in terms of their positions in this diagram in Sect. 5. In the same section, the spatial distribution and the nature of the selected young stellar sources is also discussed. Finally, conclusive remarks on this study are given in Sect. 6.

\section{Observations and data reduction}

Red giants, stars close the end of their life usually have high mass-loss rates, while stars at the earliest stages of their formation are embedded into dense molecular clumps and cores. In both cases, the dust shells or disks around the objects of interest absorb almost all of the visible radiation, which is re-radiated at longer wavelengths. As a consequence, absorption decreases very rapidly with increasing wavelength (e.g., Joyce 1992; Glass 1999), the extinction coefficient at $2.2 \mu \mathrm{m}$ being approximately $10 \%$ of that at $500 \mathrm{~nm}$. Here we are interested in young stellar sources associated with circumstellar dusty shells or disks, characterized by bright IR excess emission.

\subsection{Observations}

The near-IR images of NGC 346/N66 are obtained within the ESO Program ID 63.I-0329 (PI: M. Rubio) with the Infrared Spectrometer And Array Camera (ISAAC), mounted on the Nasmyth-Focus B of UT1 (Antu) at the Very Large Telescope (VLT). The images were taken between 24 and 26 Sep. 1999, under fair seeing conditions ( $F W H M$ between $0.6^{\prime \prime}$ and $\left.1.0^{\prime \prime}\right)$. They were used partly to detect YSOs in the region of N66 (Simon et al. 2007), but have never been presented in their complete spatial and wavelength coverage. The observations were performed with the short wavelength arm of ISAAC, using the $1024 \times 1024$ HgCdTe Hawaii Rockwell array. The pixel scale of the Hawaii detector is $0.1484^{\prime \prime} /$ pixel, providing a maximum field-of-view of $152 \times 152 \mathrm{arcsec}^{2}$. The images were obtained in the filters $J$ $(1.25 \mu \mathrm{m}), H(1.65 \mu \mathrm{m})$, and $K_{\mathrm{s}}(2.16 \mu \mathrm{m})$.

At wavelengths longer than $\sim 2.2 \mu \mathrm{m}$, the thermal background is dominated by atmospheric and telescope emission, leading to a highly variable sky brightness in the infrared. Detector cosmetics and instabilities also illustrate the need for an accurate sky subtraction. This is achieved by mean of 

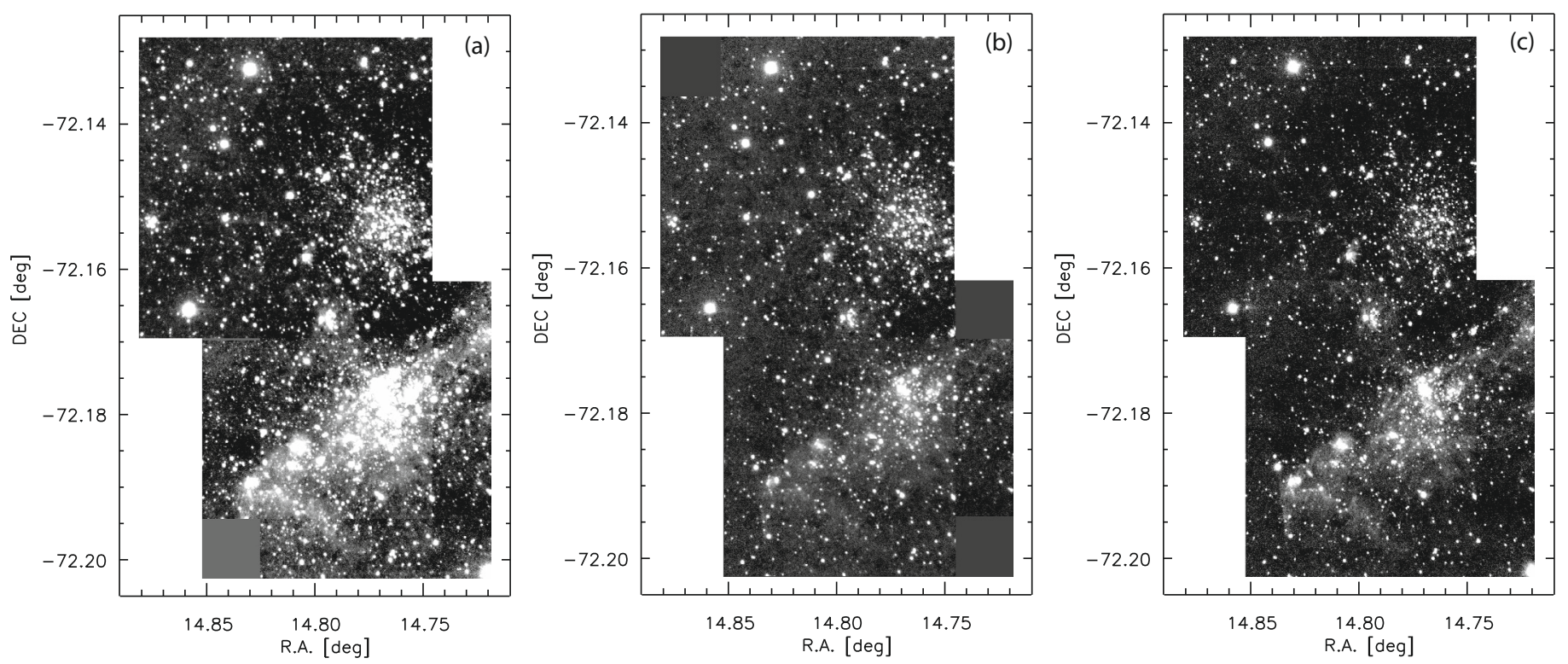

Fig. 1. Mosaic images from the combination of the northern and center jittered ISAAC frames of the area of NGC $346 / \mathrm{N}$ 66 in the a) $J$-; b) $H$-; and c) $K_{\mathrm{s}}$-band.

the technique of jittering $^{1}$, which is available for the shortwavelength and some long-wavelength imaging templates of ISAAC. A set of 10 to 100 frames are combined to form one final "jittered" frame. At the beginning and end of each night, twilight flats are taken for each filter. The following morning, dark images of the detector are taken for all detector integration times (DITs) used during the previous night. The area of NGC 346 was divided into two jittered frames, each consisting of 10 single frames. An additional jittered frame was taken for a control field of the galaxy, located to the south of the system. For each single frame, the total integration time was $60 \mathrm{~s}$, taken in 5s (DIT) exposures of 12 sub-integrations (NDIT). The fieldof-view is about $2.5^{\prime} \times 2.5^{\prime}$, corresponding to about $44 \times 44 \mathrm{pc}^{2}$ at the SMC (distance from us $60.6 \mathrm{kpc}$; Hilditch et al. 2005). We retrieved the dataset of these observations, including the science frames, twilight flats, dark images, and standard stars from the ESO Science Archive Facility ${ }^{2}$.

\subsection{Data reduction}

To reduce the data, we used ESO's ISAAC pipeline, which is based entirely on the libraries of the data reduction package ECLIPSE $^{3}$. The package consists of the main eclipse-library and the additional instrument specific pipeline packages. Another algorithm of ECLIPSE is the jitter routine, which implements efficient filtering and processing methods for infrared data reduction. The detector pixels have intrinsically different sensitivities because of the quantum efficiency variations from pixel to pixel. There is also a readout gradient of the array, which itself is not homogeneously illuminated by the telescope. These effects, as well as artifacts caused by dust on the optical surface, are corrected within the data reduction process.

The dark current of the ISAAC Hawaii array is low, so the detector bias, also called the "zero level offset", is the dominant feature of the dark frames. A master dark, constructed with

\footnotetext{
${ }^{1}$ http://www.eso.org/projects/dfs/papers/jitter99/

2 http://archive.eso.org/cms/

${ }^{3}$ http://www. eso.org/eclipse
}

ECLIPSE, was subtracted from the science frames. The effects of dust, the inhomogeneity of the illumination, and pixel to pixel variations were then removed using a master flat-field frame. Since no flat-field screen exists at UT1, for ISAAC only twilight flats are available. For the subtraction of both sky and bias, the darkest twilight flat frame was used. After this subtraction, the remaining 15 twilight flats were combined to produce a master flat-field with ECLIPSE, which also creates a bad pixel map output file. The jitter routine in ECLIPSE was then used to reduce the science data for the frames observed with acceptable seeing, by filtering out low-frequency sky variations from the set of jittered images, a method called sky combination. Two sets of jittered images were combined for the whole area of NGC 346. The corresponding final frames are shown for each filter in Fig. 1. The association NGC 346, the numerous young compact star clusters located in the bright HII-region N 66, and the intermediate-age star cluster BS 90 are easily identified in these images. The photometry, discussed in the following section, was performed on the single jittered frames for each filter. The derived catalogs were then matched to produce the complete photometric catalog of the observed regions.

\section{Photometry}

Our photometry was performed within the Image Reduction and Analysis Facility (IRAF) system ${ }^{4}$, with the package DAOPHOT ${ }^{5}$ (Stetson 1987). The effect of both the quality of the imaging system and the seeing conditions on the obtained magnitudes of the stars is quantified by the point spread function (PSF), which represents the image of a typical point source in the observations. DAOPHOT provides accurate PSF photometry of crowded stellar fields. The magnitudes of all stars detected in the observed jittered images are defined relative to the brightness of Vega. We first have to specify the input parameters of DAOPHOT to optimize the detection and photometry of stars, before using the routine daofind to detect all stellar sources in the science frames.

\footnotetext{
${ }^{4}$ http://iraf.noao.edu/

5 http://iraf.noao.edu/scripts/irafhelp?daophot
} 

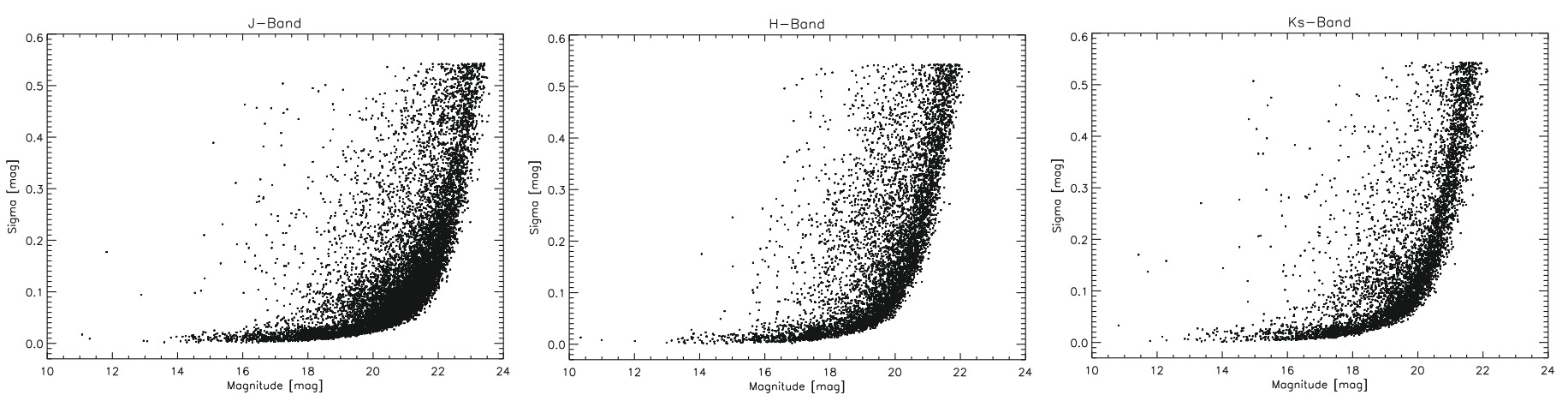

Fig. 2. Typical photometric uncertainties in the three $J, H$, and $K_{\mathrm{s}}$ bands, derived by DAOPHOT for all detected sources.

The routine phot is then used to perform aperture photometry of the detected stars and determine their instrumental magnitudes. The radius of the aperture is selected to be between 1 and 2 times that of the FWHM, depending on the effective seeing. For individual stars, the aperture radius can be up to 5 or more of that of the FWHM.

The typical PSF of our images was constructed by interactively selecting approximately 20 isolated bright stars per frame using DAOPHOT pstselect. The PSF was modelled to be the sum of an analytic bivariate Gaussian function and empirical corrections from the best Gaussian of the true observed brightness values within the average profile of several stars in the image. This process and the computation of the PSF to be fitted was performed by the routine psf. PSF photometry was performed with the routine allstar, which after classifying the stars in groups, compiles a catalog of the most likely candidate stars, based on their PSF fitting and the physical conditions, and subtracts them from the original image. The photometric process was repeated for the subtracted frames and the magnitudes of its newly discovered stars were determined. Approximately $30 \%$ more stars were found in the region of NGC 346 from the second photometry run, but most of them have large photometric uncertainties. The final numbers of identified stars in each filter is 11900 in $J, 6406$ in $H$, and 5837 in $K_{\mathrm{s}}$. We match these photometric catalogs with a procedure developed in IDL to identify stars in common. After selecting the stars with the highest photometric quality, i.e., stars with photometric uncertainties equal or smaller than $0.1 \mathrm{mag}$, we identified 2783 stars in both the $J$ and $H$ filters, 3067 stars in $J$ and $K_{\mathrm{s}}, 2550$ stars in $H$ and $K_{\mathrm{s}}$, and 2506 stars in all three filters collectively.

\subsection{Completeness and photometric accuracy}

Typical uncertainties in our photometry are shown in Fig. 2. The completeness of our photometry is evaluated by artificial star experiments, by adding artificial stars into the original frames with the DAOPHOT subroutine addstar. A few hundred artificial stars were added to each observed frame for every filter. This process was repeated several times, and the completeness of our photometry was derived by applying the photometric process to each new frame and comparing the final stellar catalogs with those of the original artificial stars based on the coordinates of the stars. The derived completeness of our photometry is shown in Fig. 3 for all three bands. Based on the completeness measurements and the photometric uncertainties, the limiting magnitudes of our photometry are $J \simeq 22.0 \mathrm{mag}, H \simeq 20.5 \mathrm{mag}$, and $K_{\mathrm{s}} \simeq 20.5 \mathrm{mag}$.

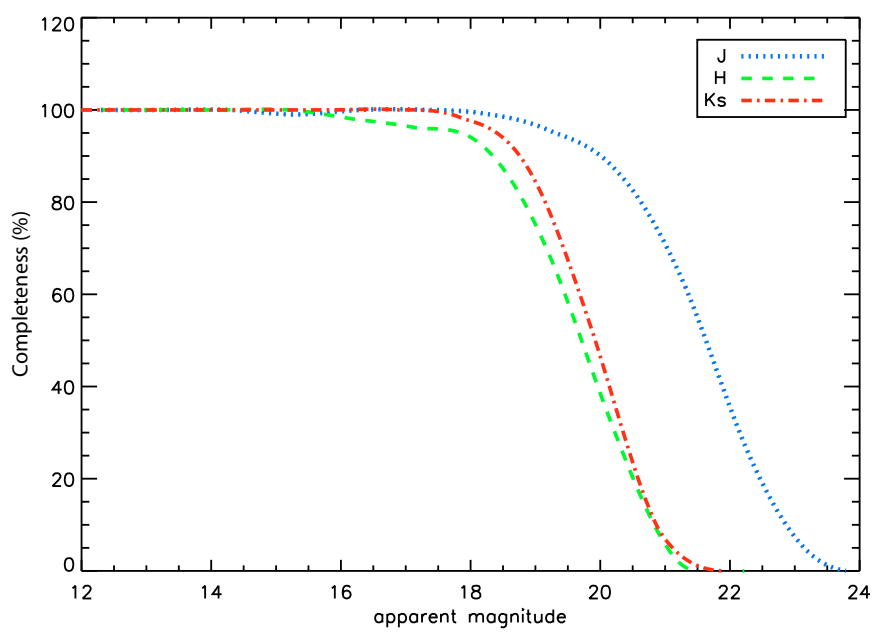

Fig. 3. Completeness for all stars detected in the three observed fields in all three $J, H$ and $K_{\mathrm{s}}$ filters.

\subsection{Photometric calibration}

We calibrate the magnitudes of the stars with near-IR absolute photometry of the same field from the Two Micron All Sky Survey $^{6}$ (2MASS). The 2MASS filter system agrees very well with that of ISAAC, and the 2MASS Point Source Catalog (AllSky 2003) is therefore particularly suitable for the calibration of our photometry. The application $\mathrm{ALADIN}^{7}$ was used to identify all stars found with 2MASS in the same field-of-view as ours and to compare the magnitudes measured from both ISAAC and 2MASS data sets. To calculate the magnitude offsets between the two photometries, we selected more than 20 bright stars with the highest quality photometric data from our photometric cata$\log$ in each filter. The comparison of our photometry with that of 2MASS is shown in Fig. 5. The offsets per filter are calculated in terms of the median of the differences between the magnitudes of the stars, which are common to both ISAAC and 2MASS samples. The apparent magnitude offsets are $1.812 \mathrm{mag}$ in $J, 0.678 \mathrm{mag}$ in $H$, and $0.367 \mathrm{mag}$ in $K_{\mathrm{s}}$. The uncertainties in this calibration can be quantified by the standard deviations derived from the dispersion in 2MASS-ISAAC magnitudes for the common stars (Fig. 5 - bottom). They are $\sigma_{J}=0.14 \mathrm{mag}$, $\sigma_{H}=0.12 \mathrm{mag}$, and $\sigma_{K_{\mathrm{s}}}=0.13 \mathrm{mag}$, respectively.

\footnotetext{
${ }^{6}$ http://irsa.ipac.caltech.edu

7 http://aladin.u-strasbg.fr/aladin.gml
} 
Table 1. Sample of the photometric catalog of stars found in this study in the region of NGC 346/N66 and a nearby control field in all three $J, H$, and $K_{\mathrm{s}}$ filters with VLT ISAAC imaging.

\begin{tabular}{rcccccccccc}
\hline \hline ID & $\begin{array}{c}\text { R.A. } \\
(\mathrm{deg})\end{array}$ & $\begin{array}{c}\text { Decl. } \\
(\mathrm{deg})\end{array}$ & $\begin{array}{c}X \\
(\mathrm{px})\end{array}$ & $\begin{array}{c}\mathrm{C} \\
(\mathrm{px})\end{array}$ & $\begin{array}{c}J \\
(\mathrm{mag})\end{array}$ & $\begin{array}{c}\sigma_{J} \\
(\mathrm{mag})\end{array}$ & $\begin{array}{c}H \\
(\mathrm{mag})\end{array}$ & $\begin{array}{c}\sigma_{H} \\
(\mathrm{mag})\end{array}$ & $\begin{array}{c}K_{\mathrm{s}} \\
(\mathrm{mag})\end{array}$ & $\begin{array}{c}\sigma_{K_{\mathrm{s}}} \\
(\mathrm{mag})\end{array}$ \\
\hline 1 & 14.79218 & -72.16765 & 531.765 & 155.835 & 16.855 & 0.013 & 16.853 & 0.012 & 16.920 & 0.023 \\
2 & 14.79437 & -72.16741 & 548.261 & 150.011 & 16.011 & 0.014 & 15.968 & 0.013 & 15.979 & 0.034 \\
3 & 14.85005 & -72.16258 & 965.954 & 31.829 & 17.483 & 0.007 & 17.098 & 0.010 & 17.097 & 0.018 \\
4 & 14.79519 & -72.16639 & 554.363 & 124.946 & 17.957 & 0.029 & 18.110 & 0.054 & 18.113 & 0.043 \\
5 & 14.79583 & -72.16637 & 559.162 & 124.505 & 15.665 & 0.004 & 15.664 & 0.009 & 15.731 & 0.018 \\
6 & 14.79584 & -72.16676 & 559.283 & 134.180 & 18.141 & 0.025 & 17.840 & 0.037 & 17.714 & 0.055 \\
7 & 14.80519 & -72.16749 & 629.400 & 151.956 & 18.724 & 0.023 & 18.307 & 0.026 & 18.074 & 0.027 \\
8 & 14.81704 & -72.16776 & 718.270 & 158.700 & 19.061 & 0.017 & 18.626 & 0.031 & 18.561 & 0.033 \\
9 & 14.84279 & -72.16213 & 911.515 & 20.758 & 17.483 & 0.006 & 17.117 & 0.010 & 17.097 & 0.016 \\
10 & 14.85175 & -72.16175 & 978.702 & 11.646 & 19.181 & 0.021 & 19.364 & 0.065 & 19.252 & 0.059 \\
11 & 14.85197 & -72.16337 & 980.320 & 51.276 & 18.321 & 0.010 & 17.905 & 0.022 & 17.907 & 0.023 \\
12 & 14.78242 & -72.16232 & 458.576 & 25.405 & 17.969 & 0.014 & 17.820 & 0.019 & 17.878 & 0.023 \\
13 & 14.78620 & -72.16421 & 486.962 & 71.662 & 16.760 & 0.008 & 16.605 & 0.009 & 16.645 & 0.011 \\
14 & 14.79341 & -72.16464 & 541.046 & 82.180 & 18.041 & 0.008 & 17.567 & 0.016 & 17.488 & 0.014 \\
15 & 14.80191 & -72.16452 & 604.786 & 79.288 & 18.263 & 0.011 & 17.814 & 0.019 & 17.679 & 0.017 \\
\hline
\end{tabular}

Notes. This table is available in its entirety at the CDS.

\subsection{The photometric catalog}

We derived celestial coordinates for all stars detected with high quality photometry $(\sigma \leq 0.1 \mathrm{mag}$ ) from their pixel coordinates in the final jitter FITS images and used the applications xy2sky and sky2xy available from the World Coordinate Systems (WCS) Tools ${ }^{8}$ (ver. 3.7.2). We transformed the $(X, Y)$ coordinates of the stars into celestial coordinates according to the astrometric corrections provided in the FITS header of the corresponding jittered images by applying xy2sky. We then transformed the celestial coordinates of all stars with the use of sky $2 x y$ into a common $(X, Y)$ pixel coordinate system with respect to the central field, where NGC $346 / \mathrm{N} 66$ is mainly observed, by using the astrometric corrections provided in the FITS header of the $K_{\mathrm{s}}$ image of this field. A sample of the final compiled catalog of the stars found in all three filters is given in Table 1. This table is available in its entirety at the CDS. In Fig. 4, the map of all sources detected in all three wavebands is shown.

\section{Observed stellar populations}

In our subsequent analysis, we consider only sources detected with the highest quality photometric parameters and with photometric uncertainties based on the PSF fitting of $\sigma \leq 0.1$ mag in every waveband. The region NGC 346/N66 is known to host a mixture of stellar populations (e.g., Gouliermis et al. 2006), including the evolved SMC field stars, the young main-sequence (MS) and pre-main sequence (PMS) populations of the association NGC 346 and its vicinity (e.g., Hennekemper et al. 2008), and the 4.5 Gyr-old faint MS and bright RGB stars of the cluster BS 90 (Rochau et al. 2007).

\subsection{Color-magnitude diagrams}

The color-magnitude diagrams (CMDs) were compiled from our photometry for all stars detected in both the area of NGC 346/N66 (where BS 90 is also included) and the control field, specifically the $J-H$ versus (vs.) $H, H-K_{\mathrm{s}}$ vs. $K_{\mathrm{s}}$, and $J-K_{\mathrm{s}}$ vs. $K_{\mathrm{s}}$ CMDs, shown in Fig. 6 . The variety of stellar types in the observed region is illustrated by the superimposed isochrone models. These evolutionary models, which

\footnotetext{
8 Available at http://tdc-www. harvard.edu/wcstools/
}

are designed for the ESO Imaging Survey WFI $U B V R I Z$ and SOFI $J H K$ VEGAmag systems, were developed by Girardi et al. (2002). The superimposed isochrones, corresponding to ages $\sim 4$ Myr and $\sim 4.5$ Gyr, were selected to correspond to the metallicity of the SMC, namely $Z=0.004$ (Haser et al. 1998; Bouret et al. 2003). Both the bright MS of NGC 346 and the red giant branch (RGB) of the field and BS 90 are clearly defined in the CMDs of Fig. 6, these features being the most clearly distinguished in the $J-K_{\mathrm{s}}, K_{\mathrm{s}} \mathrm{CMD}$. To determine the interstellar reddening of the observed stellar populations, we assume a Galactic interstellar extinction law

$R_{V}=\frac{A_{V}}{E_{B-V}}$

where $R_{V}$ is roughly equal to $3.14 \pm 0.10$ (Schultz \& Wiemer 1975). The Galactic extinction in near-IR wavebands is given by $A_{J}=0.282 \times A_{V}, A_{H}=0.175 \times A_{V}$ and $A_{K}=0.112 \times A_{V}$ (Rieke \& Lebofsky 1985). Assuming these factors, we found that to fit the young isochrone to the observed bright MS population in the CMDs of Fig. 6, an extinction of $A_{V} \simeq 0.3$ mag should be applied. On the other hand, the observed RGB stars do not appear to be affected by any significant reddening in the CMDs ( $A_{V} \simeq 0.02 \mathrm{mag}$ ). This implies that the younger MS stars are still embedded within dust, while the older population of the field and BS 90 are not located in regions of high nebulosity, i.e., BS 90 is located in front of N66, and consequently their light does not suffer from extinction.

\subsection{Variations in the color-magnitude diagram}

The stellar systems NGC 346 and BS 90 represent two quite different types of clusters both from morphological and evolutionary point-of-views. Their evolutionary difference is naturally based on the stellar member populations of each system, which should define the prominent features in the corresponding CMDs of the systems. This becomes clearer when the CMDs of selected areas centered on these systems, comprising the most representative stellar populations in the systems, are constructed. In Fig. 7, the $J-K_{\mathrm{s}}, K_{\mathrm{s}}$ CMDs of (a) a circular area of radius $\sim 35^{\prime \prime}$ (10 pc) centred on NGC 346, and (b) a circular area of radius $\sim 0.5^{\prime}(8.5 \mathrm{pc})$ centred on BS 90 are shown. These radii do not correspond to the sizes of the systems, but are selected to be very close to their centers to isolate the most prominent 


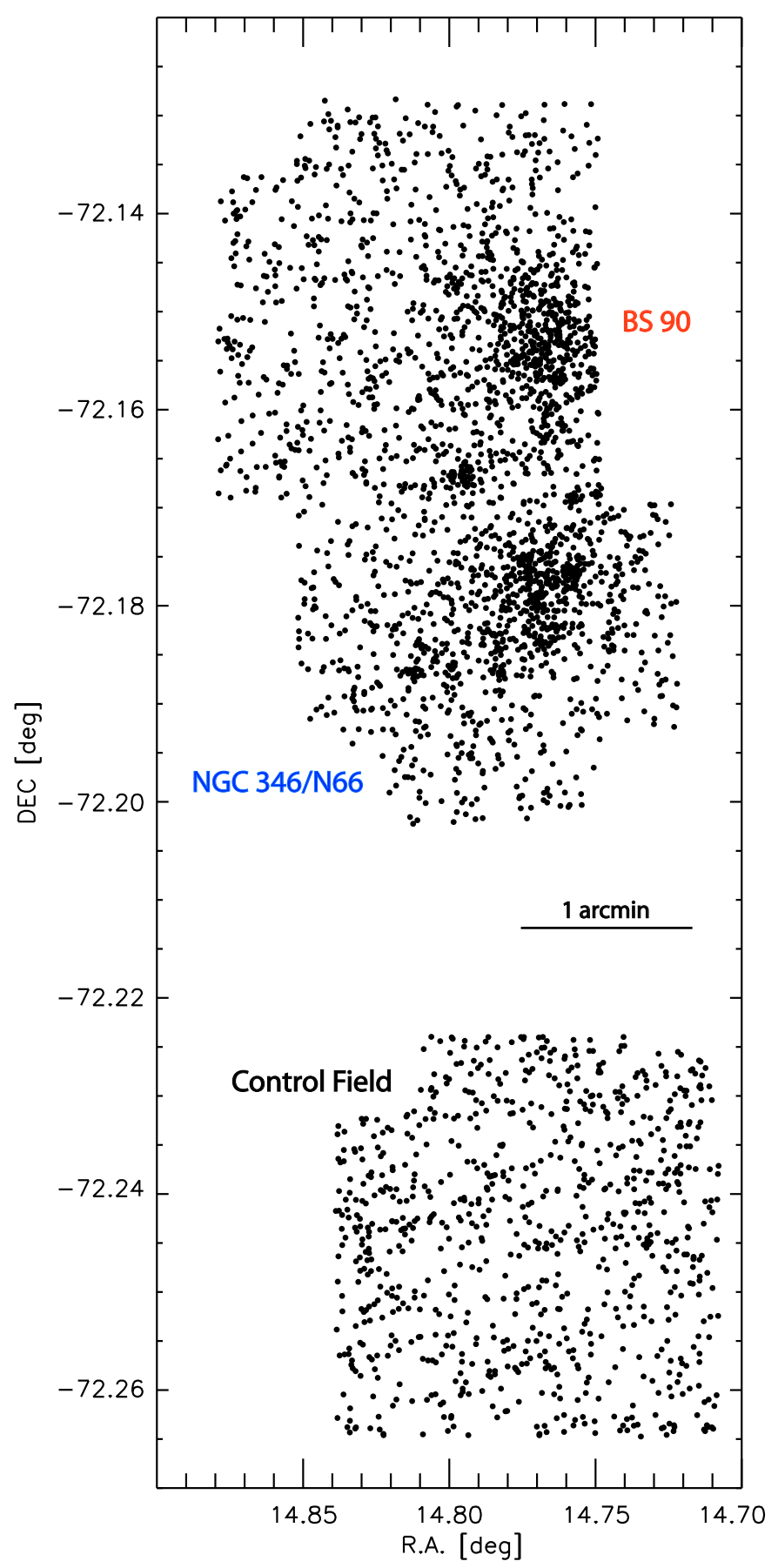

Fig. 4. Chart of all stars detected with good photometric accuracy in all three filters in all three fields observed with VLT/ISAAC. This map shows the relative positions of the observed fields: The northern field that includes the intermediate-age cluster BS 90, the central field, where the association NGC 346 and the main part of the H II region N66 are located, and the remote control-field, which covers the representative stellar populations of the general SMC field in the area. North is up and East is to the left of the map.

stellar populations in each system. The corresponding CMD of the control field is also shown for comparison (Fig. 7c).

The variations in the CMD from one area to the next are indicated by the superimposed isochrone models in Fig. 7. The stellar population of the star-forming association NGC 346, as seen in the CMD of Fig. 7a, includes mostly young bright MS stars with a small contamination of the general field of the SMC, as

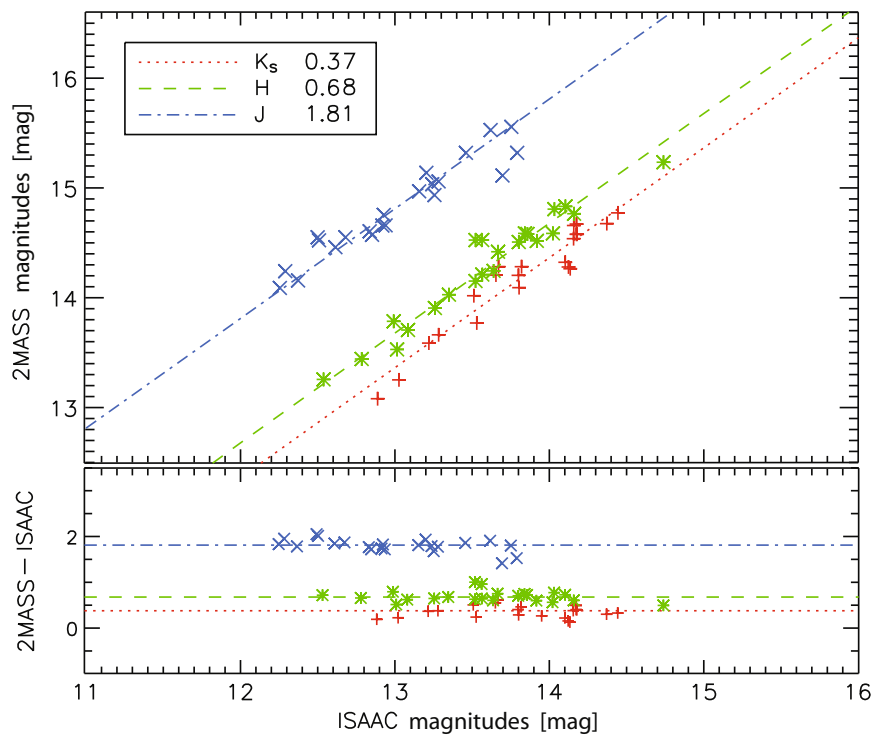

Fig. 5. Comparison of our photometry with that derived by 2MASS for more than 20 common stars, located in all three observed fields for all three $J, H, K_{\mathrm{s}}$ filters. Top: ISAAC versus (vs.) 2MASS photometry for the common stars. The linear fits that define the offset for each filter are shown with different lines and colors. The corresponding offsets applied to the absolute calibration of our photometry are also given for every filter. Bottom: Dispersion in the 2MASS-ISAAC magnitudes for the common stars as a function of ISAAC magnitudes. The offsets for each filter are again defined by different lines. The standard deviation in the points derived from the corresponding mean values is the measurement of the uncertainties in our photometric calibration for each filter (see Sect. 3.2).

this is defined by the CMD of the control field shown in Fig. 7c. Typical positions of early-type stars are also shown in Fig. 7a based on the study of Hanson et al. (1997). On the other hand, the stellar content of the intermediate-age star cluster BS 90, seen in the CMD of Fig. 7b, is characterized by the prominent RGB and few supergiants of age $\sim 4.5$ Gyr. The positions of late-type giants are also plotted based on Peletier (1989) and Binney \& Merrifield (1998). Isochrone fitting on the CMDs of Fig. 7 allows us to derive an indicative age for the stellar systems in the region. For NGC 346, we find an age $~ 3.9 \mathrm{Myr}$, consistent with that found by, e.g., Nota et al. (2006). The CMD on BS 90 is most accurately fitted by the isochrone of an age $4.5 \mathrm{Gyr}$, in good agreement with the value previously found by Sabbi et al. (2007) and Rochau et al. (2007). It should be noted that BS 90 is a populous cluster, of large spatial extent, and therefore the contamination of the stellar populations observed in the area of the association NGC 346 by those of BS 90 should not be negligible. We discuss this in detail in Sect. 5.1. Finally, the CMD of the control field provides evidence of a variety of stellar populations, which are the results of different star formation events, of a wide range of ages, from young MS populations with age $\sim 30 \mathrm{Myr}$, an order of a magnitude older than those in NGC 346, to evolved giants of age $\gtrsim 2 \mathrm{Gyr}$. We note that near-R photometry of early-type MS stars is quite insensitive to ages. Therefore, the ages given here are only indicative.

The bright main sequence stars in NGC 346/N66 are known to be O-, B-, or early A-type stars of masses between 3 and $60 M_{\odot}$, while its low- and intermediate-mass stellar content is characterized by a large sample of pre-main sequence (PMS) stars (e.g., Hennekemper et al. 2008). The most massive of these stars, such as Herbig Ae/Be stars, are possibly also located in the 

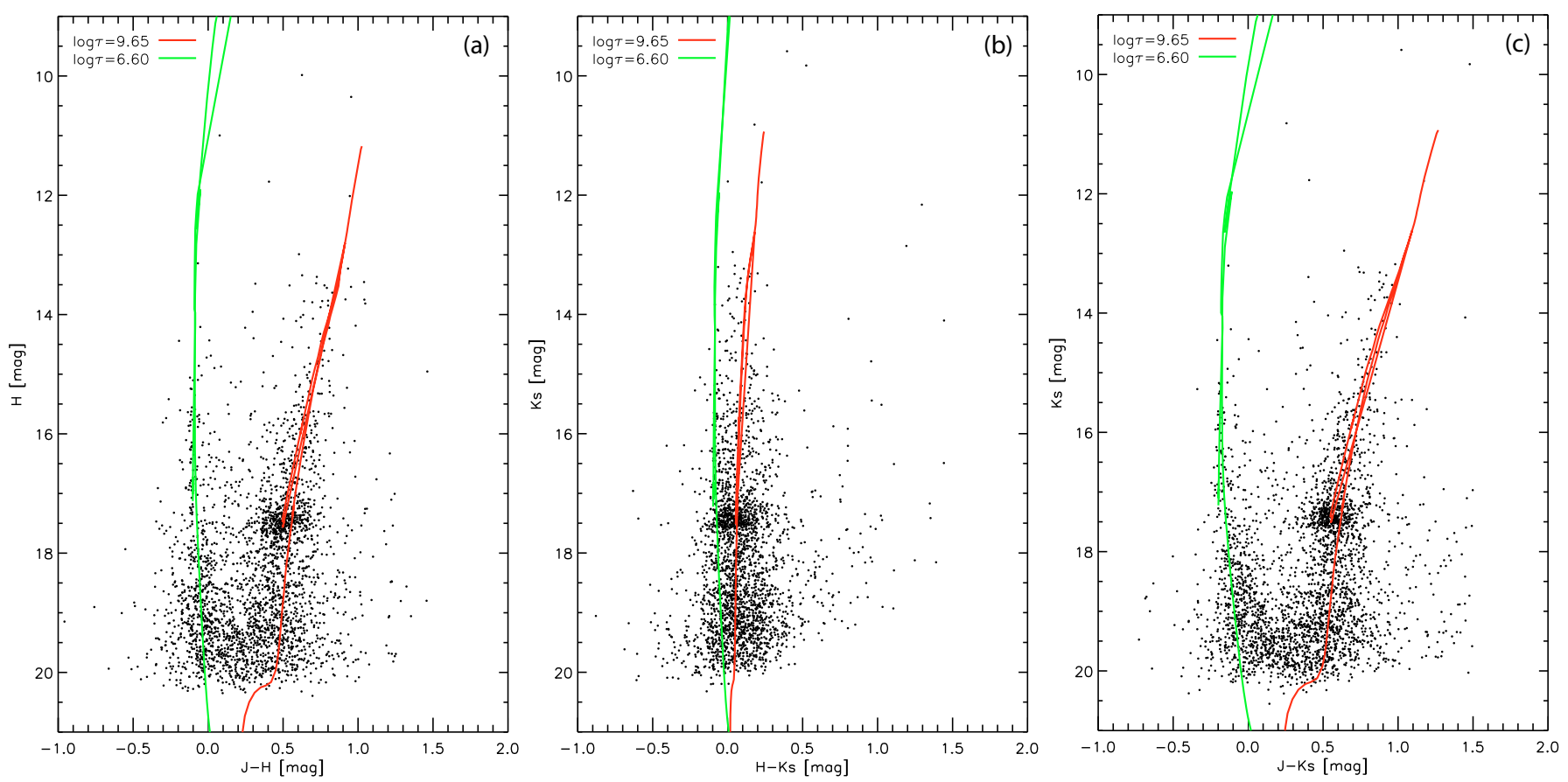

Fig. 6. CMDs of all stars found in all observed fields of the region NGC 346/N66 and its nearby control field of the SMC. CMDs in different combinations of the three bands are shown. Specifically, a) $J-H, H$; b) $H-K_{\mathrm{s}}, K_{\mathrm{s}}$; and c) $J-K_{\mathrm{s}}, K_{\mathrm{s}}$. Two indicative isochrones from the Padova grid of evolutionary models (Girardi et al. 2002), corresponding to ages $\sim 4 \mathrm{Myr}$ and $\sim 4.5 \mathrm{Gyr}$ are over-plotted to demonstrate the differences of the comprised stellar populations.

red part of the main-sequence of Fig. 7a. However, our photometry is not in general deep enough to detect significant numbers of low-mass PMS stars. The part of the CMD between the MS and the RGB should also host classical Be stars (see e.g., Bik et al. 2006). To perform a more accurate identification of these sources and to utilize the near-IR three-band color-color diagram of the detected sources in the young association NGC 346, we focus our subsequent analysis on the central area of NGC 346/N66.

\section{Stellar sources under formation}

There are no comparable studies to ours in the SMC, but there have been a few previous near-IR photometric investigations of resolved populations in star-forming regions of the lowmetallicity environment of the Large Magellanic Cloud (LMC). A combination of ground-based near-IR data and space optical observations of the 30 Dor Nebula by Rubio et al. (1998) helped identify numerous stellar IR sources in or near the bright nebular filaments west and northeast of R136, suggesting that a new stellar generation is being produced by the energetic activity of the massive central cluster in the remanent interstellar material around its periphery. Near-IR photometry of the second largest HII region in the LMC, N11B, also highlighted several bright IR sources (Barbá et al. 2003). Several of these sources have IR colors with YSO characteristics, and they are prime candidates to be intermediate-mass Herbig Ae/Be (HAeBe) stars.

Deep near-IR imaging of the N159/N160 star-forming region in the LMC detected candidate HAeBe stars down to $\sim 3 M_{\odot}$, based on their near-IR colors and magnitudes (Nakajima et al. 2005). Two embedded HAeBe clusters were also discovered, one of them, and two neighboring $\mathrm{H}$ II regions, providing hints of the beginning of sequential cluster formation in N159S. The spatial distributions of the $\mathrm{HAeBe}$ and $\mathrm{OB}$ clusters indicate largescale sequential cluster formation over the entire observed region from N160 to N159S. Near-IR photometry obtained to study the stellar content of the LMC star-forming region N4 is used to study the connection between the interstellar medium and its stellar content (Contursi et al. 2007). This analysis found several bright IR sources with characteristics of massive, early O-type stars. However, according to these authors, IR spectroscopy of these sources would confirm their very young and massive nature.

Chen et al. (2009) presented the most complete identification of YSO candidates in the LMC H II complex N44. These authors combined mid- and far-IR Spitzer Space Telescope imaging with complementary ground-based imaging in $U B V I J K$ to classify the YSOs into Types I, II, and III, according to their spectral energy distributions (SEDs). In their sample, $\sim 65 \%$ of these objects were resolved into multiple components or extended sources. The distribution of the YSO candidates compared with those of the underlying stellar population and interstellar gas illustrates a correlation between the current formation of O-type stars and previous formation of massive stars, providing evidence of triggered star formation in N44.

In connection to the aforementioned studies, our photometry, although not very deep, provides original near-IR colors of candidate young sources in the vicinity of N66. However, from nearIR photometry alone it is impossible to accurately determine the nature of the most IR-bright sources, unless this photometry is part of a multi-band optical and IR investigation, as we discuss later. In the present study, we provide only a first set of accurate near-IR colors for all young objects in the region, and select the most interesting IR-bright objects from our photometric catalog, based on their near-IR colors and their near-IR excess inferred from the $J-H, H-K_{\mathrm{s}}$ color-color diagram (C-CD). This selection is described in the following section.

\subsection{Selection of young stellar sources}

To identify the sources that represent the most recent star formation in the region, we first consider the contribution of both 

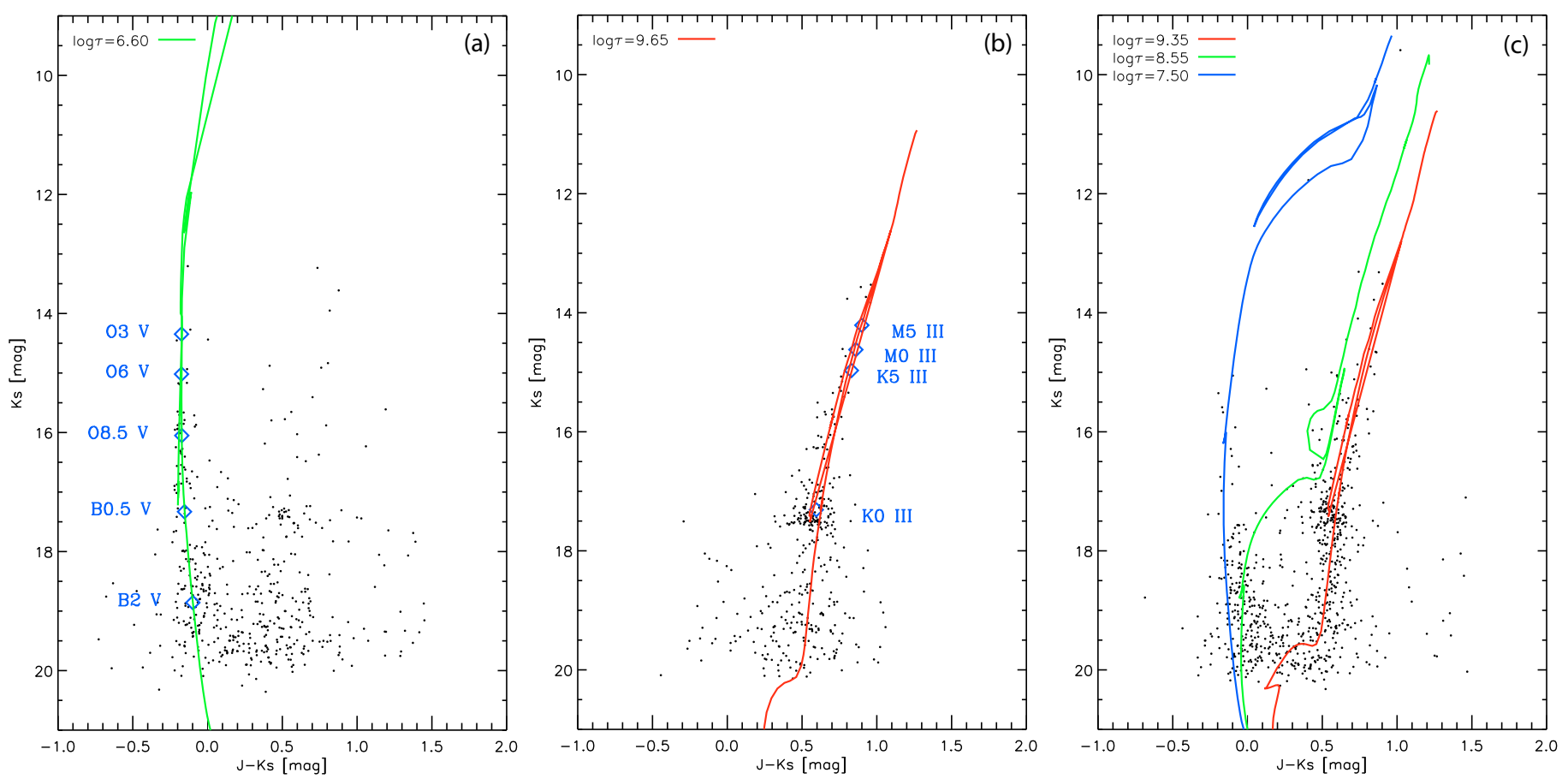

Fig. 7. $J-K_{\mathrm{s}}, K_{\mathrm{s}}$ CMDs of stars found in two selected parts of the observed region NGC 346/N66, for a) a circular area of radius $\sim 10$ pc centered on NGC 346 ; b) a circular area of radius $\sim 8.5 \mathrm{pc}$ centered on BS 90; and c) the control field alone. The variations in the CMD from one area to another are highlighted by the superimposed isochrone models and typical positions of early-type MS stars and late-type giants (see Sect. 4.2).

the evolved MS and RGB stellar populations of the SMC field, the cluster BS 90, and the association NGC 346 to the complete stellar sample. As shown in Figs. 6 and 7, the MS is well defined with its brighter members belonging to the association, but it is its red part and the region between the MS and RGB that host stellar sources at their earlier stages of formation (see e.g., Bik et al. 2006). Therefore, and to eliminate the significant contamination of this part of the CMD by the general field and BS 90, which is very close to the central part of N66, we constrain our analysis to the ISAAC FOV that covers the main body of NGC 346/N66, defined as the central field with $-72.21<\operatorname{Dec}(\mathrm{deg}) \leq-72.15$ in the map of Fig. 4. Colorcomposite images of this field, which covers the association NGC 346, the bar of N66, and several subclusters and HII regions in its vicinity, are shown in Fig. 8. The dust emission, seen in the $8 \mu \mathrm{m}$ band, indicates the centers of the most recent star formation and demonstrates the different information revealed by different wavelengths for the same region.

The use of the near-IR C-CD is a reliable method for detecting sources, which are characterized by near-IR excess emission, from their positions in the diagram. In this diagram, the evolved populations are easily identified by comparison with models and considering interstellar extinction, PMS stars, namely classical and weak-line T Tauri (CTT, WTT) stars (e.g., Appenzeller \& Mundt 1989), Herbig Ae/Be (HAeBe) stars (e.g., Perez \& Grady 1997; Waters \& Waelkens 1998), and other YSOs (e.g., Lada \& Wilking 1984; André 1994) are located in the reddest part. This is a signature of their IR excess due to their circumstellar dust in the form of cocoons or disks. Hence, when selecting candidate YSOs and PMS stars, we consider only the sources that are detected in the central ISAAC field in all three $J H K_{\mathrm{s}}$ wavebands. The $J-K_{\mathrm{s}}, K_{\mathrm{S}} \mathrm{CMD}$ and $J-H, H-K_{\mathrm{S}} \mathrm{C}-\mathrm{CD}$ of these sources are shown in Fig. 9. Considering that BS 90 to be a large cluster with a tidal radius between $r_{\mathrm{t}} \simeq 2$ '.15 (Sabbi et al. 2007) and 3!'15 (Rochau et al. 2007), its spatial extent covers the central field of
NGC 346/N66, and consequently affects the stellar content of the region. This is clearly demonstrated in the CMD of Fig. 9, where a prominent RGB can be seen. While these stars are bright in the near-IR, they do not exhibit any significant excess emission. Our classification to identify young stellar sources based on their near-IR excess, therefore allows us to effectively discard most of the evolved stellar contaminants from our sample of selected young sources in formation. However, a definite selection requires a sophisticated multi-band study on an individual source basis, as we discuss in Sect. 5.2.

In the $J-K_{\mathrm{s}}$ vs. $K_{\mathrm{S}} \mathrm{CMD}$ of Fig. 9, the areas occupied by HAeBe (Eiroa et al. 2002) and classical Be stars (Dougherty et al. 1994) are delineated by two ellipses, and the positions of massive Galactic YSOs identified by Hanson et al. (1997) and Bik et al. (2006) are indicated by green stellar symbols. In the C-CD of Fig. 9, the typical positions of T Tauri (TTS), HAeBe, and classical Be stars are drawn to indicate the loci, where these sources in our sample should be expected in this diagram. We note, however, that typical TTS are fainter than the detection limit of our photometry and therefore are barely discernible in the CMD of Fig. 9, and only a few of them being seen in the C$\mathrm{CD}$. We refer, however, to these PMS stars for reasons of completeness. The diagrams of Fig. 9 appear to comprise a large variety of different stellar types at quite different evolutionary stages. The regions in the CMD and C-CD, where classical Be stars are typically expected, also include MS populations. Searching for Be stars, Keller et al. (1999) found in six fields centered on young clusters of the Magellanic Clouds (NGC 346 included), that the average fraction of $\mathrm{Be}$ to normal $\mathrm{B}$ stars is similar to that found in the Galaxy ( 20\%, see, e.g., Cox 2000, p. 414). No connection between the Be star fraction and age or metallicity was found by these authors, and the classical Be stars detected in NGC 346 do not have any influence on the evolution of the region. According to their near-IR colors, red giants and subgiants are expected, to occupy the blue edges of the typical locations of 

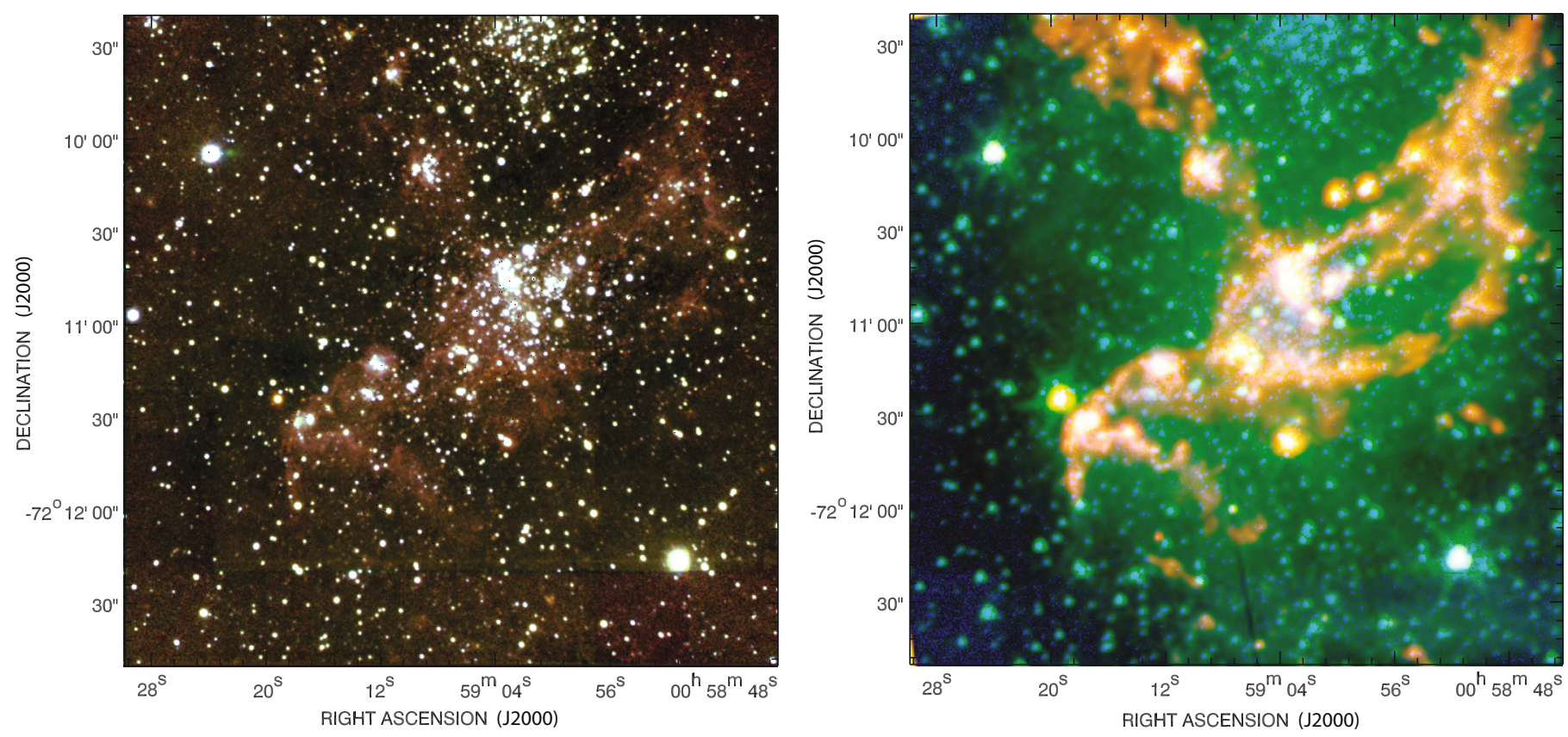

Fig. 8. Color-composite images of the main part of the region NGC 346/N66. Left: Image constructed with the combination of the jittered images in $J$ (blue), $H$ (green), and $K_{\mathrm{s}}$ (red) from VLT/ISAAC imaging. Right: Image produced from the $K_{\mathrm{s}}$ ISAAC image (blue) and Spitzer/IRAC $3.6 \mu \mathrm{m}$ and $8 \mu \mathrm{m}$ filters (green and red respectively). ISAAC images were constructed with DIMSUM (Deep Infrared Mosaicing Software), an external package of IRAF, especially designed to produce accurate sky subtracted images from dithered observations.
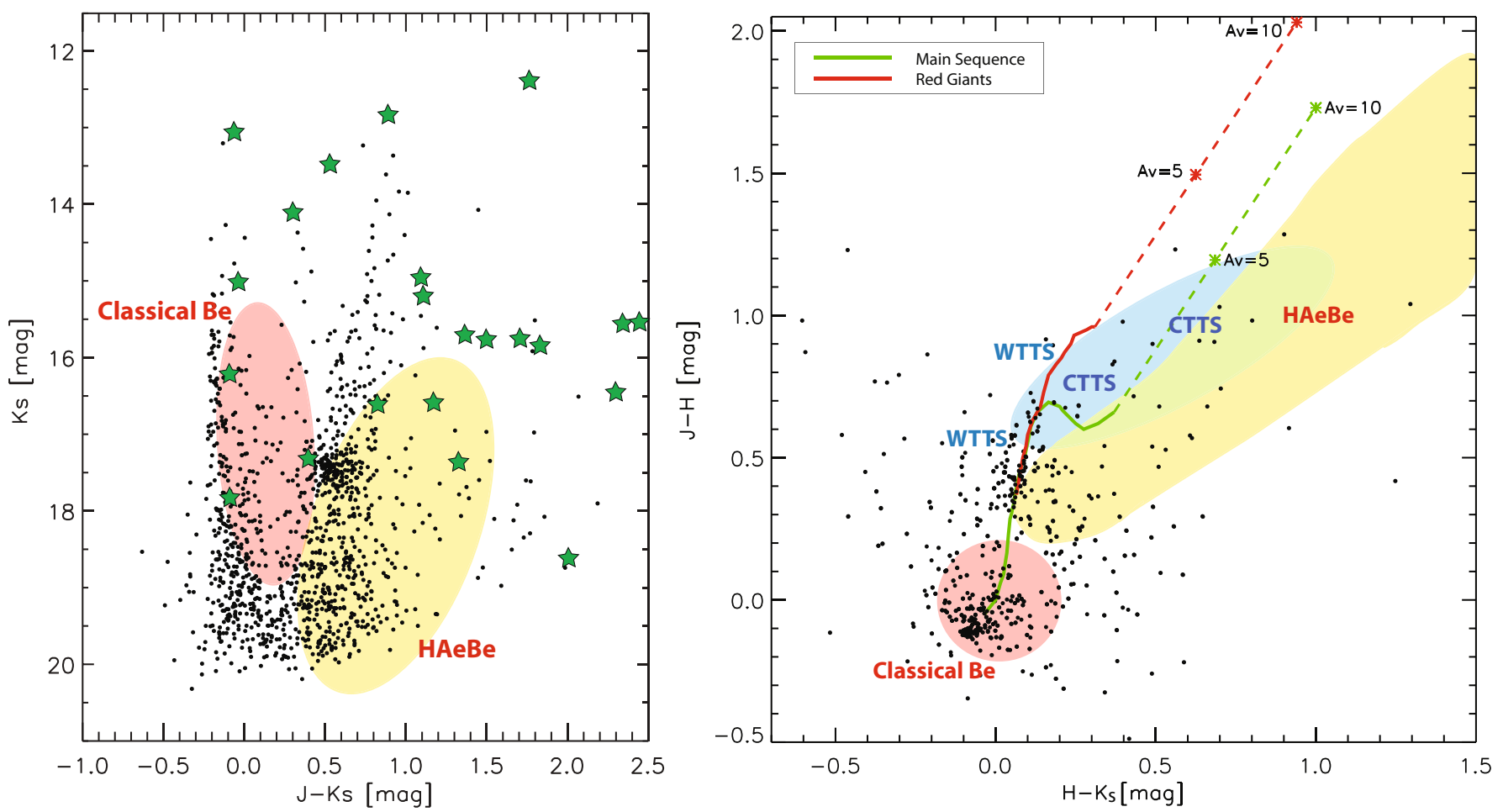

Fig. 9. $J-K_{\mathrm{s}}$ vs. $K_{\mathrm{s}}$ color-magnitude diagram (CMD) and the corresponding $J-H$ vs. $H-K_{\mathrm{s}}$ color-color diagram (C-CD) of all sources found in the main part of the star-forming region NGC 346/N66 with high photometric accuracy ( $\sigma \lesssim 0.1 \mathrm{mag}$ ) from our VLT/ISAAC photometry. Typical locations of Herbig Ae/Be stars (HAeBe; yellow shaded areas) and classical Be stars (red shaded areas) are shown based on the studies by Lada \& Adams (1992), Dougherty et al. (1994), Meyer et al. (1997) and Eiroa et al. (2002). While T Tauri stars (TTS) are fainter than our detection limit in the CMD, typical positions of classical T Tauri stars (CTTS; blue shaded area), and the general loci of weak-line T Tauri stars (WTTS) are also shown in the C-CD for completeness. In the CMD, the green " $\star$ " symbols represent the extinction-corrected positions of the massive YSOs from the samples of Hanson et al. (1997) and Bik et al. (2006). The green and red lines in the C-CD denote the loci of MS and RGB stars, respectively. Reddening vectors for $A_{\mathrm{V}}=5 \mathrm{mag}$ and $10 \mathrm{mag}$ are also drawn. These diagrams can be reliably utilized to distinguish the sources with infrared excess located to the right of the reddening vectors, away from the evolved MS and RGB stars. 

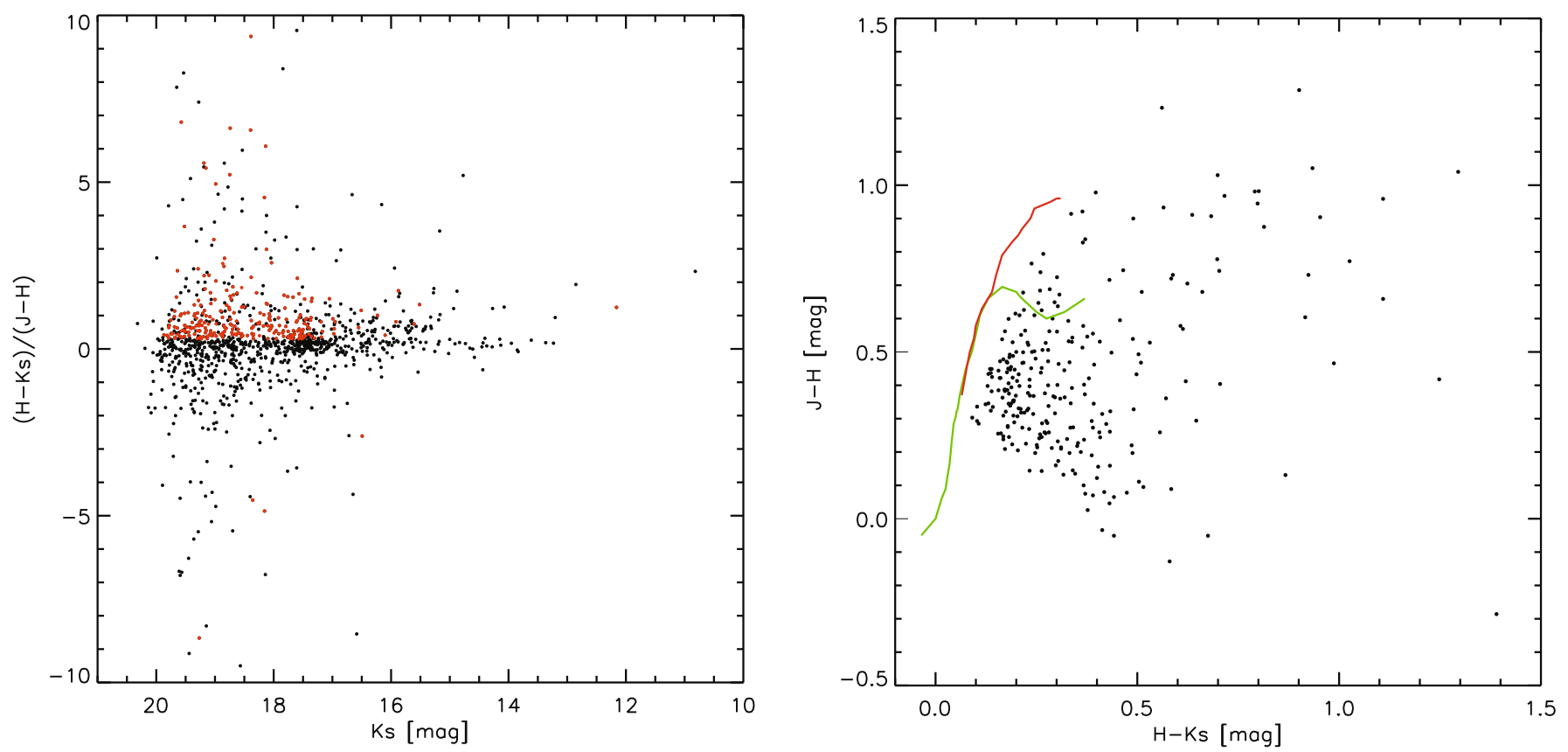

Fig. 10. Selection of the sources identified to have a near-IR excess based on their locations in the $H-K_{\mathrm{s}}, J-H \mathrm{C}-\mathrm{CD}$, and $J-K_{\mathrm{s}}, K_{\mathrm{s}} \mathrm{CMD}$ (see Fig. 9). Left: The fraction of the color indices $\left(H-K_{\mathrm{s}}\right) /(J-H)$ as a function of the $K_{\mathrm{s}}$ magnitudes of the detected sources. Right: The C-CD diagram of all sources selected as the most prominent young stellar candidates with near-IR excess emission.

HAeBe stars. As a consequence, we should be careful to differentiate the sources that are most probably at their earlier stages of evolution, based on their near-IR excess, from the evolved red stars of the region.

Based on the discussion above, we make a first tentative selection of the sources that most probably have near-IR excess using a diagram of the color fraction $\left(H-K_{\mathrm{s}}\right) /(J-H)$ of all detected objects as a function of their brightness in $K_{\mathrm{s}}$. In this diagram, shown in Fig. 10 (left), one can see that most sources are located along a horizontal sequence of stars with $\left(H-K_{\mathrm{s}}\right) /(J-H) \simeq 0.0$, corresponding to the RGB and MS stars that exhibit no near-IR excess. Young stellar sources with a near-IR excess should be located away from the horizontal sequence. We first assume that all sources with $\left(H-K_{\mathrm{s}}\right) \lesssim 0.0$ should not have any near-IR excess, as they are located blueward of the MS in the C-CD of Fig. 9. We then apply a first-order selection of the remaining sources based on the criterion that an absolute offset from the horizontal sequence in the color fraction $\left(H-K_{\mathrm{s}}\right) /(J-H)$ of about 0.3 is a reasonable limit to separate the stars with near-IR excess from those that show no excess. As a consequence, we select as sources with near-IR excess those that have color fractions $\left(H-K_{\mathrm{s}}\right) /(J-H) \geq 0.3$ or $\left(H-K_{\mathrm{s}}\right) /(J-H) \leq-0.3$ in the diagram of Fig. 10 (left).

However, an investigation of the positions of these nearIR bright sources in the CMD of Fig. 9 shows that there is significant contamination of these objects by classical Be and RGB stars, which should be eliminated. Therefore, we place yet tighter constraints on our sample by selecting only the sources that fall at the positions expected for HAeBe stars as shown in Fig. 9 (left) and redder. More precisely, we select the sources that fulfill the following criteria: (i) They are located to the blue part of the MS in the C-CD with $\left(H-K_{\mathrm{S}}\right)>0.0$. (ii) They have fractions of color indices $\left(H-K_{\mathrm{s}}\right) /(J-H) \geq+0.3$ or $\leq-0.3$. (iii) They are located to the red part of a diagonal line that tangentially crosses the blue edge of the HAeBe area, specified in the $J-K_{\mathrm{s}}, K_{\mathrm{s}}$ CMD of Fig. 9. These sources represent our final sample of objects selected as the most likely star-forming candidates, and the subject of our analysis here. The positions of these sources are indicated by red points in the diagram $\left(H-K_{\mathrm{s}}\right) /(J-H)$ vs. $K_{\mathrm{s}}$ of Fig. 10 . Their positions in the C$\mathrm{CD}$ are also shown in Fig. 10 (right panel). In this $\mathrm{C}-\mathrm{CD}$, it is indeed evident that our selected sample probably corresponds to objects with strong near-IR excess emission.

\subsection{The sample of candidate young stellar sources in NGC 346/N66}

The selection scheme returned 263 candidate young stellar sources in the main part of the region NGC 346/N66. The positions of these sources are shown in a map of this area in Fig. 11 as circular blue points. They are superimposed on a colorcomposite image constructed by combining our ISAAC image in the $K_{\mathrm{S}}$ filter (green component) with archived images obtained with the Infrared Array Camera (IRAC; Fazio et al. 2004) onboard the Spitzer Space Telescope and in particular channel 4 (8.0 $\mu \mathrm{m}$, red component), within the GTO science program 63 (PI: J. R. Houck). These IRAC data were used to detect candidate YSOs in the general region of N66 by Simon et al. (2007). The objects identified by these authors as "definite" YSOs are also plotted in Fig. 11 with large red circular symbols.

In this map the positions of the detected candidate YSOs in NGC 346/N66 clearly correspond to dusty structures seen in the $8 \mu \mathrm{m}$ emission as red filaments, these objects being located at peaks of mid-IR emission in these filaments. These peaks can also be seen - at lower spatial resolution - in the $24 \mu \mathrm{m}$ band of Multiband Imaging Photometer for SIRTF (MIPS; e.g. Heim et al. 1998; Rieke et al. 2003) onboard Spitzer, and they are also observed in the $2.14 \mu \mathrm{m} \mathrm{H}_{2}$ line and the ISOCAM LW2 band that covers 5-8 $\mu \mathrm{m}$ (Contursi et al. 2000; Rubio et al. 2000). We note that the spatial distribution of our candidate young stellar sources follows the filamentary dust emission, being concentrated along a few southern dusty arcs (at the middle and bottom of the map) and one northern dusty arm (at the top of the 


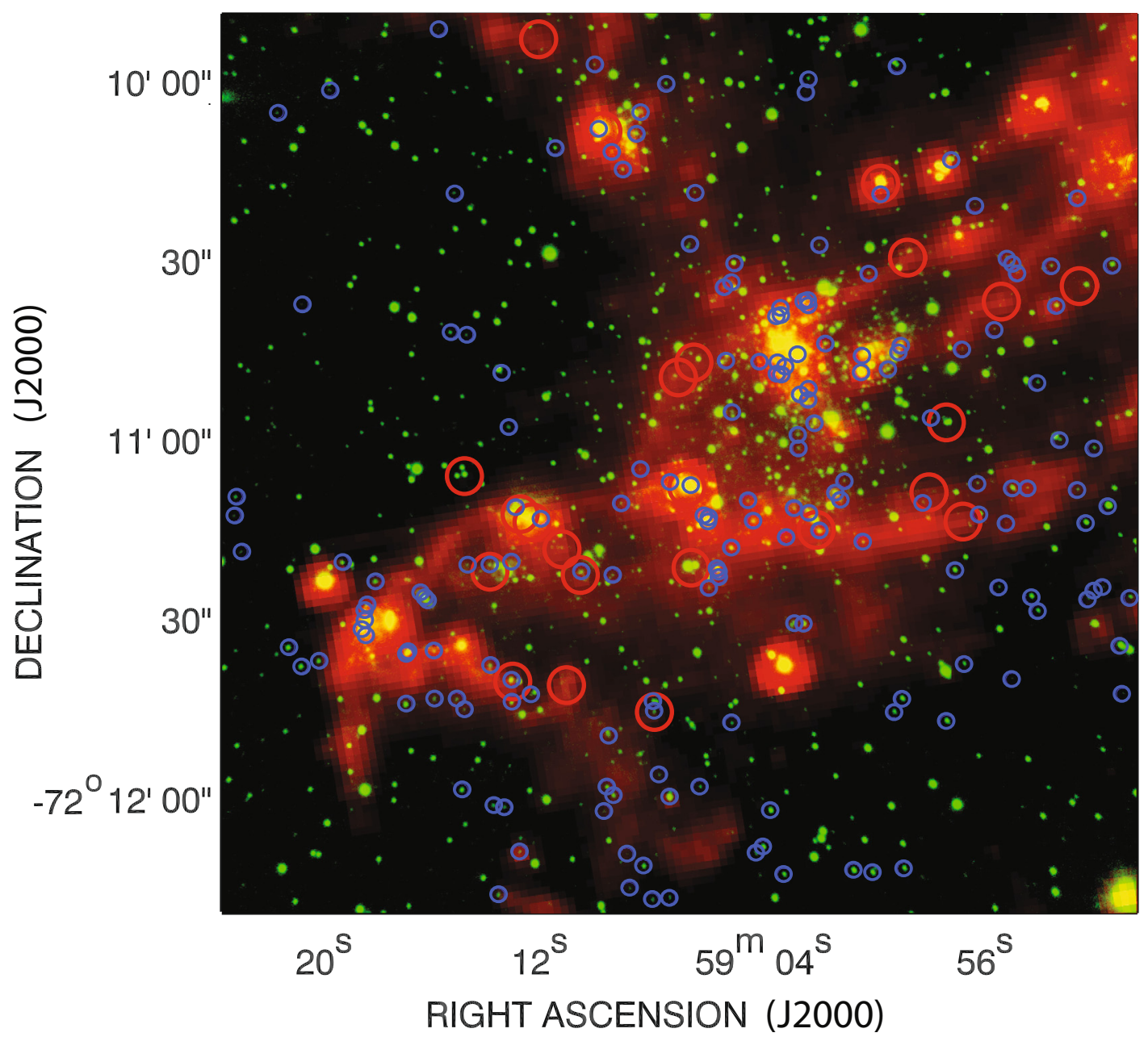

Fig. 11. Positions of stellar sources in our photometric catalog, with strong near-IR excess emission, based on their positions in the $J-H, H-K_{\mathrm{s}}$ C-CD and $J-K_{\mathrm{s}}, K_{\mathrm{S}}$ CMD (see Fig. 9). They are probably young stellar sources in the process of formation such as intermediate-mass PMS stars and high-mass YSOs. Their positions are overplotted with blue circle symbols on a color-composite image consisting of the $K_{\mathrm{s}}$ ISAAC image (green) and Spitzer/IRAC $8 \mu \mathrm{m}$ filters (red). Candidate YSOs from the catalog of Simon et al. (2007) are overplotted with large red circular symbols.

image). The latter, along with the south-eastern filament, is understood to be the product of a more recent triggered star formation event (Gouliermis et al. 2008). Hennekemper et al. (2008) discuss the spatial distribution of the low-mass PMS stars detected by Gouliermis et al. (2006), and of sources with excess $\mathrm{H} \alpha$ emission that are the most likely candidates to be intermediatemass PMS stars (their Figs. 1 and 2). The spatial distribution of all these objects follows the same trend as our sources, and it is probable that both catalogs include several objects in common. In addition, the positions of a significant number of candidate young stellar sources found in our near-IR photometry, shown in Fig. 11, also seem to be quite clustered in several concentrations. We also note that almost all of these concentrations coincide with subclusters of PMS stars observed with HST/ACS identified by applying two cluster analysis techniques (Schmeja et al. 2009).

We first compared a nominal search-box of $2^{\prime \prime}$ of the cata$\log$ of our sources with that of the YSOs found with Spitzer by Simon et al. (2007) and PMS stars with $\mathrm{H} \alpha$ excess found with Hubble by Hennekemper et al. (2008) in the same region. This comparison returns more than $\sim 40 \%$ of the $\mathrm{H} \alpha$ excess stars and $\sim 60 \%$ of the YSOs also detected in the near-IR by ourselves. There are cases where more than one objects found with Hubble coincide with one of our sources, while in other cases a few of our sources are found as components of one of the YSOs found with Spitzer within the specified search-box. This phenomenon is caused by differences in the resolving efficiency of the three instruments, demonstrating the importance of resolving multiple sources into their true components and classifying their true nature. The resolution achieved by VLT/ISAAC is at least 10 times higher than that of Spitzer/IRAC, allowing a reasonable identification of any components in multiple YSOs. However, 15\% of our sources coinciding with $\mathrm{H} \alpha$-excess objects identified by Hennekemper et al. (2008), are resolved by HST/ACS to be multiple systems themselves. Therefore, the differences in resolution between the data sets obtained with HST, VLT, and Spitzer is a crucial issue in identifying true single-objects, or the components of multiple systems in our sample. As we discuss later, to perform a more detailed study in the near-IR, observations of the highest possible spatial resolution are essential.

It is almost certain that our sample of candidate PMS stars and YSOs is incomplete, since for example TTS are not included because of our detection limit. Other young stellar sources such as HAeBe stars may also be missing due to the strong constraints of our selection criteria. In addition, our sample may be contaminated by evolved stars, which in general do not exhibit 
any significant near-IR excess. While the positions of most of the sources in our catalog coincide with the dust filaments of N66, illustrating their youthfulness, there are candidate young sources in our sample that are located away from the dusty filaments of Fig. 11. These are usually assumed to be evolved stars, or even background galaxies, rather than stellar sources in formation. However, decontaminating our sample of old stars in the field and BS 90 located in the central region of NGC 346/N66 on a statistical basis for such a small sample, or based on their positions away from the dust filaments, will possibly compromise the catalog of sources with true near-IR excess and it produce selection effects.

A more sophisticated selection on a source-by-source basis is certainly required to identify the most prominent objects that represent the most recent star formation in the region. This, however, can only be achieved through the excessive use of imaging in many different wavebands and/or spectroscopy (e.g., Chen et al. 2009), so that complete SEDs of individual sources can be constructed and consequently their true nature accurately defined (e.g., Whitney et al. 2008). Observations of higher spatial resolution will allow the components of any unresolved bright sources in our sample to be recognized. Such a thorough analysis would certainly include the use of previous observations of the region NGC 346/N66 from various telescopes at different wavelengths including those of HST (Gouliermis et al. 2006; Hennekemper et al. 2008) and Spitzer (Bolatto et al. 2007; Simon et al. 2007), as well as new near-IR observations of higher spatial resolution and sensitivity. A preliminary study of the available data and the preparation of follow-up observations in the near-IR is currently being performed by ourselves.

\section{Conclusions}

We have presented a detailed near-IR photometric study with VLT/ISAAC of the star-forming region NGC 346/N66 in the SMC. We have used archival ISAAC imaging of the general area of N66, which includes the stellar association NGC 346, the intermediate-age cluster BS 90, and a southern empty control field of the SMC. We have performed photometry on images obtained in the filters $J(1.25 \mu \mathrm{m}), H(1.65 \mu \mathrm{m})$, and $K_{\mathrm{s}}$ $(2.16 \mu \mathrm{m})$ and derived a catalog of more than 2500 stars detected in all three wavebands. The color-magnitude diagrams of these stars include a collection of different stellar populations, comprising the young MS stars of the association mixed with the RGB and old MS stars of BS 90 and the general field of the SMC, but also objects that are at their earlier stages of their formation. We select the best PMS and YSO candidates in our sample on the basis of their positions in the near-IR color-color and color-magnitude diagrams.

We focus the selection of these sources on the central field observed with ISAAC, which covers only the main part of the nebula N66 and the association NGC 346 to avoid any severe contamination of our sample with the evolved red stars of BS 90 and the field. In this area, our photometry detected 1174 stars in all three wavebands for which the near-IR CMD and C-CD are constructed. In these diagrams, the evolved stellar populations are mostly aligned along the sequences of RGB and MS stars as they are expected to be by the evolutionary models depending on the interstellar extinction, but certainly contaminate the sample of young stellar sources. The reason is that, while these sources, such as low-mass T Tauri stars, intermediate-mass Herbig Ae/Be stars, and massive YSOs, exhibit excess emission in the near-IR due to circumstellar dust, their positions in the CMD and C-CD do not necessarily cover the reddest part.
Bearing this in mind, we make a tentative selection of PMS and YSO candidates with $\left(H-K_{\mathrm{S}}\right) \gtrsim 0.0$ (redder than MS and $\mathrm{RGB})$ in the diagram of the color fraction $\left(H-K_{\mathrm{s}}\right) /(J-H)$ as a function of the $K_{\mathrm{s}}$ brightness. In this diagram, sources that have an excess in their near-IR colors are located away from the horizontal sequence of evolved stars. We select the sources that have an offset from the horizontal sequence of $\mid \Delta\left(H-K_{\mathrm{s}} / J-\right.$ $H) \mid \geq 0.3$ and we decontaminate the sample by selecting sources located in the area of the $J-K_{\mathrm{s}}, K_{\mathrm{s}}$ CMD that HAeBe stars are expected to occupy and with redder colors. We consider the selected objects as the most probable candidates to be stars in formation. This selection delivers 263 candidate young stellar sources, which are located along the dusty filamentary structures of N66 seen in the $8 \mu \mathrm{m}$ emission from Spitzer and the $2.14 \mu \mathrm{m}$ $\mathrm{H}_{2}$ line.

Combining observations at several wavelengths to construct complete SEDs of individual sources is the most accurate means of establishing their true nature. Objects from our catalog of young stellar sources do indeed coincide with candidate YSOs detected with Spitzer and sources with excess emission in $\mathrm{H} \alpha$ in the region observed with Hubble. However, since a large amount of data per object is required for detailed SED studies, it is necessary to enhance the available data sets with new data, preferably obtained with cameras of higher resolving power, so that multiple objects can be resolved in their components.

Acknowledgements. We thank M. Rubio and R. H. Barbá for their comments and suggestions. D.A.G. kindly acknowledges the support by the Deutsche Forschungsgemeinschaft (DFG) through grant GO 1659/1-2. This research has made use of the SIMBAD database, operated at the CDS, Strasbourg, France, of NASA's Astrophysics Data System, and images obtained with the Spitzer Space Telescope, which is operated by the Jet Propulsion Laboratory, California Institute of Technology under a contract with NASA. It is also based on observations made with ESO Telescopes at the La Silla Paranal Observatory under program ID 063.I-0329.

\section{References}

André, P. 1994, in The Cold Universe, ed. T. Montmerle, C. J. Lada, I. F. Mirabel, \& J. Tran Thanh van, 179

Appenzeller, I., \& Mundt, R. 1989, A\&ARv, 1, 291

Barbá, R. H., Rubio, M., Roth, M. R., \& García, J. 2003, AJ, 125, 1940

Bica, E. L. D., \& Schmitt, H. R. 1995, ApJS, 101, 41

Bik, A., Kaper, L., \& Waters, L. B. F. M. 2006, A\&A, 455, 561

Binney, J., \& Merrifield, M. 1998, Galactic astronomy, ed. J. Binney \& M. Merrifield

Bolatto, A. D., Simon, J. D., Stanimirović, S., et al. 2007, ApJ, 655, 212

Bouret, J.-C., Lanz, T., Hillier, D. J., et al. 2003, ApJ, 595, 1182

Chen, C.-H. R., Chu, Y.-H., Gruendl, R. A., Gordon, K. D., \& Heitsch, F. 2009, ApJ, 695, 511

Contursi, A., Lequeux, J., Cesarsky, D., et al. 2000, A\&A, 362, 310

Contursi, A., Rubio, M., Sauvage, M., Barba, D. C. R., \& Boulanger, F. 2007, A\&A, 469, 539

Cox, A. N. 2000, Allen's astrophysical quantities, ed. A. N. Cox

Dougherty, S. M., Waters, L. B. F. M., Burki, G., et al. 1994, A\&A, 290, 609

Eiroa, C., Oudmaijer, R. D., Davies, J. K., et al. 2002, A\&A, 384, 1038

Evans, C. J., Lennon, D. J., Smartt, S. J., \& Trundle, C. 2006, A\&A, 456, 623

Fazio, G. G., Hora, J. L., Allen, L. E., et al. 2004, ApJS, 154, 10

Girardi, L., Bertelli, G., Bressan, A., et al. 2002, A\&A, 391, 195

Glass, I. S. 1999, Handbook of Infrared Astronomy, ed. I. S. Glass

Gouliermis, D. A., Dolphin, A. E., Brandner, W., \& Henning, T. 2006, ApJS, 166,549

Gouliermis, D. A., Chu, Y.-H., Henning, T., et al. 2008, ApJ, 688, 1050

Hanson, M. M., Howarth, I. D., \& Conti, P. S. 1997, ApJ, 489, 698

Haser, S. M., Pauldrach, A. W. A., Lennon, D. J., et al. 1998, A\&A, 330, 285

Heim, G. B., Henderson, M. L., Macfeely, K. I., et al. 1998, in SPIE Conf. Ser. 3356, ed. P. Y. Bely, \& J. B. Breckinridge, 985

Henize, K. G. 1956, ApJS, 2, 315

Hennekemper, E., Gouliermis, D. A., Henning, T., Brandner, W., \& Dolphin, A. E. 2008, ApJ, 672, 914 
D. A. Gouliermis et al.: ISAAC imaging of the SMC star-forming region NGC 346/N66

Hilditch, R. W., Howarth, I. D., \& Harries, T. J. 2005, MNRAS, 357, 304

Joyce, R. R. 1992, in Astronomical CCD Observing and Reduction Techniques, ed. S. B. Howell, ASP Conf. Ser., 23, 258

Keller, S. C., Wood, P. R., \& Bessell, M. S. 1999, A\&AS, 134, 489

Kennicutt, Jr., R. C. 1984, ApJ, 287, 116

Lada, C. J. \& Adams, F. C. 1992, ApJ, 393, 278

Lada, C. J., \& Kylafis, N. D. 1991, The physics of star formation and early stellar evolution

Lada, C. J., \& Wilking, B. A. 1984, ApJ, 287, 610

Massey, P., Parker, J. W., \& Garmany, C. D. 1989, AJ, 98, 1305

Meyer, M. R., Calvet, N., \& Hillenbrand, L. A. 1997, AJ, 114, 288

Nakajima, Y., Kato, D., Nagata, T., et al. 2005, AJ, 129, 776

Nota, A., Sirianni, M., Sabbi, E., et al. 2006, ApJ, 640, L29

Peletier, R. F. 1989, PhD Thesis, University of Groningen, The Netherlands, (1989)
Perez, M. R., \& Grady, C. A. 1997, Space Sci. Rev., 82, 407

Rieke, G. H., \& Lebofsky, M. J. 1985, ApJ, 288, 618

Rieke, G. H., Young, E. T., Alonso-Herrero, A., et al. 2003, BAAS, 35, 1243

Rochau, B., Gouliermis, D. A., Brandner, W., Dolphin, A. E., \& Henning, T. 2007, ApJ, 664, 322

Rubio, M., Barbá, R. H., Walborn, N. R., et al. 1998, AJ, 116, 1708

Rubio, M., Contursi, A., Lequeux, J., et al. 2000, A\&A, 359, 1139

Sabbi, E., Sirianni, M., Nota, A., et al. 2007, AJ, 133, 44

Schmeja, S., Gouliermis, D. A., \& Klessen, R. S. 2009, ApJ, 694, 367

Schultz, G. V., \& Wiemer, W. 1975, A\&A, 43, 133

Simon, J. D., Bolatto, A. D., Whitney, B. A., et al. 2007, ApJ, 669, 327

Stetson, P. B. 1987, PASP, 99, 191

Walborn, N. R., Lennon, D. J., Heap, S. R., et al. 2000, PASP, 112, 1243

Waters, L. B. F. M., \& Waelkens, C. 1998, ARA\&A, 36, 233

Whitney, B. A., Sewilo, M., Indebetouw, R., et al. 2008, AJ, 136, 18 